\title{
Manipulation and the zygote argument: Another reply
}

Markus E. Schlosser, University College Dublin, markus.schlosser@ucd.ie Forthcoming in The Journal of Ethics

This is the author's copy (which may differ from the final print version in minor details)

\begin{abstract}
Alfred Mele's zygote argument is widely considered to be the strongest version of the manipulation argument against compatibilism (about free will and determinism). Opponents have focused largely on the first of its two premises and on the overall dialectic. My focus here will be on the underlying thought experiment - the Diana scenario - and on the second premise of the argument. I will argue that reflection on the Diana scenario shows that the second premise does not hold, and we will see that my objection to the second premise helps to defend the claim that manipulation arguments face, in general, a dilemma.
\end{abstract}

Keywords: Manipulation arguments, zygote argument, compatibilism, free will, moral responsibility

\section{Introduction: The zygote argument}

Manipulation arguments are supposed to show that free will and moral responsibility are incompatible with determinism by showing that there is no relevant difference between being causally determined to perform an action and being manipulated to do it. They are, that is, supposed to provide an argument for incompatibilism and against compatibilism (see Kane 1996, Fischer \& Ravizza 1998, Pereboom 2001, and Mele 2006, for instance). ${ }^{1}$ The most recent debate on this has focused on Alfred Mele's zygote argument, which is widely considered to be the

\footnotetext{
${ }^{1}$ Arguably, traditional compatibilism supports a response strategy to this challenge that is not available to proponents of the currently prominent non-traditional versions of compatibilism (such as Frankfurt 1971, Dennett 1984, and Fischer \& Ravizza 1998). According to traditional compatibilism, the ability to do otherwise is compatible with determinism. Non-traditional versions of compatibilism deny this (or remain agnostic about this). The manipulation of an agent may bring it about that the agent will not do otherwise without bringing it about that the agent could not do otherwise. If a manipulated agent retains the ability to do otherwise, then he or she may well be free and morally responsible (partly because of having the ability to do otherwise). If, on the other hand, a manipulated agent is not able to do otherwise, then traditional compatibilists can argue that this must be due to the fact that the agent has been manipulated (rather than the fact that the agent's actions are causally determined). This move is not available to proponents of non-traditional compatibilism, because they do not hold that the ability to do otherwise is compatible with determinism. However, as traditional compatibilism is widely rejected, we can put this issue aside and limit our considerations to the currently prominent non-traditional versions of compatibilism.
} 
strongest version of the manipulation argument currently on offer. This argument is based on the following thought experiment, which features Diana, a goddess with very special powers:

Diana creates a zygote $Z$ in Mary. She combines $Z$ 's atoms as she does because she wants a certain event $E$ to occur thirty years later. From her knowledge of the state of the universe just prior to her creating $Z$ and the laws of nature of her deterministic universe, she deduces that a zygote with precisely Z's constitution located in Mary will develop into an ideally self-controlled agent who, in thirty years, will judge, on the basis of rational deliberation, that it is best to $A$ and will $A$ on the basis of that judgment, thereby bringing about $E$. [...] Thirty years later, Ernie is a mentally healthy, ideally self-controlled person who regularly exercises his powers of self-control and has no relevant compelled or coercively produced attitudes. Furthermore, his beliefs are conducive to informed deliberation about all matters that concern him, and he is a reliable deliberator. So he satisfies a version of my proposed compatibilist sufficient conditions for having freely $A$-ed. (Mele 2006: 188)

One might get distracted here by the question of whether Ernie can freely perform and be responsible for other actions (apart from $A$ ), although it is clear that Diana's creation of $Z$ is a cause of all of Ernie's actions. In order to prevent such worries, Mele asks us to assume that Diana creates the zygote with the aim of ensuring that Ernie performs all those other actions as well (2006: 190). Let us call this the Diana scenario.

According to Mele, the Diana scenario supports the first premise of the zygote argument, which says that Ernie is not a free agent and not morally responsible for anything, because of the way his zygote was produced (2006: 189). In defense of the zygote argument, Todd (2013) recently argued that proponents of the argument should drop the explanatory claim ("because of the way his zygote was produced"), because incompatibilists might want to explain and defend the core of the first premise in different ways. I am happy to accept this, as nothing depends on it for my purposes. Taking this into account, the zygote argument can be stated as follows:

(1) Ernie is not a free agent and is not morally responsible for anything.

(2) Concerning free action and moral responsibility of the beings into whom the zygotes develop, there is no significant difference between the way Ernie's zygote comes to exist and the way any normal human zygote comes to exist in a deterministic universe.

(3) Hence, determinism precludes free action and moral responsibility. 
Mele recommends that compatibilists should try to find a plausible way of denying 1 , because he sees "no way around premise 2" (2006: 193). Opponents and commentators have since devoted most of their attention to premise 1 and to the question of whether compatibilists can plausibly claim that Ernie is free and responsible. ${ }^{2}$ Fischer (2011), for instance, proposed an interesting thought experiment that raises doubts about premise 1. Kearns (2012) distinguished between a number of explanations of how, exactly, the Diana scenario is supposed to support premise 1, and he argued that none of them can be combined with premise 2 so that the conclusion follows. I largely agree with Kearns' criticisms of the argument, and I also think that it is important to consider how the two premises and the associated intuitive judgments interact. However, unlike Kearns, I propose to begin the assessment of the argument by putting premise 1 to the side and by reflecting, first, on the Diana scenario and on premise 2. In the following section, I will argue that those considerations show that premise 2 does not hold. In section 3, I turn to the overall dialectic and I will argue that my objection to premise 2 helps to defend the claim that manipulation arguments are in general bound to fail, because they face a dilemma.

\section{The Diana scenario and premise 2}

Premise 2 says that, concerning freedom and moral responsibility, there is no significant difference between the way Ernie's zygote comes to exist and the way any normal human zygote comes to exist in a deterministic universe. It says, as I shall put it, that there is no significant difference between the Diana scenario and normal deterministic scenarios.

There are, of course, some very obvious and striking differences. Most obviously, zygotes in normal deterministic scenarios are not created by some special agent who has the plan and the power to create agents so that they will perform specific actions during their development and adulthood. Premise 2 says that this striking difference is not a significant difference concerning freedom and moral responsibility. What is the argument for this claim?

According to one possible line of argument, there is no significant difference, because there is no difference in terms of common compatibilist conditions on freedom and moral responsibility. Given that the scenarios in question are deterministic scenarios, we can put aside libertarian accounts of freedom and moral responsibility and restrict our considerations to

\footnotetext{
${ }^{2}$ Two recent exceptions are Repko Waller (2014) and Barnes (forthcoming). See footnote 3.
} 
compatibilist accounts. The currently prominent compatibilist accounts feature conditions on the role of higher-order attitudes, the agent's ability to respond to reasons, the agent's ability to exercise self-control, and other conditions of this kind (see Frankfurt 1971, Dennett 1984, Fischer \& Ravizza 1998, for instance). It does not matter, here, which one of those accounts provides the best contemporary compatibilist account, and it does not matter whether any one of them provides the right account of freedom and moral responsibility. What matters, rather, is that it seems clear that the mentioned conditions may be satisfied in normal deterministic scenarios and in the Diana scenario. If this is correct, then this line of argument puts considerable pressure on the currently prominent compatibilist accounts, as it shows that those views do not have the resources to distinguish between normal deterministic scenarios and the Diana scenario. But this line of argument is not promising as an argument in support of premise 2.

Unsurprisingly, most opponents of the zygote argument hold some version of contemporary compatibilism. There is, however, no obvious reason to think that one must appeal to the claims and conditions of contemporary compatibilist accounts when one argues that there is a significant difference between normal deterministic scenarios and the Diana scenario. Being a committed proponent of a particular version of contemporary compatibilism is one thing; rejecting premise 2 of the zygote argument is another. To put this differently, the claim that there is no significant difference according to contemporary compatibilist accounts simply does not entail that there is no significant difference. It may be, instead, that contemporary compatibilist accounts are deficient precisely because they fail to capture the difference. This puts pressure on contemporary compatibilist accounts. But it does not provide an argument for premise 2.

A second line of argument can be introduced by way of raising the following rhetorical question. Ask yourself, why should a difference in how the zygote is created make any difference to freedom and moral responsibility during adulthood? This is a rhetorical question, because it is meant to evoke the obvious answer that a difference in how the zygote is created simply does not make any difference to freedom and moral responsibility during adulthood. Even some opponents of the zygote argument seem to think that this is just obvious. Fischer, for instance, says that it does "seem very clear" that how the zygote is produced has "nothing to do" with the question of whether the agent will develop into a free and morally responsible agent (Fischer 2011: 267). In support of this intuition, Mele asks us to compare the Diana scenario with a deterministic world in which Ernie's zygote is created in a normal way and in which he performs exactly the same 
actions as in the Diana scenario, on the basis of exactly the same processes of practical reasoning and reflection. As Ernie has obviously "no say" about the way in which his zygote is produced in either of the two scenarios, and as he acts in exactly the same way on the basis of exactly the same processes of practical reasoning and reflection, it seems clear that there can be no significant difference concerning freedom and moral responsibility (see Mele 2006: 190).

This line of argument is more promising. But I will now argue that it hides an important feature of the Diana scenario and that it distracts, thereby, from a striking difference between the scenarios which is also a significant difference-a difference concerning freedom and moral responsibility. This second line of argument asks us, in effect, to split the agents' histories into two parts and to consider them separately. It asks us to compare the following two issues in isolation: the way in which the zygote is created and the way in which the agent develops and acts thereafter. By hypothesis, there is no difference in the way in which the agent develops and acts, and so it seems that only a difference in the way in which the zygote is created could make a relevant difference to the agent's freedom and moral responsibility — and this seems implausible. But that is too quick, because there is a significant difference concerning how the zygote's creation is related to how the agent develops and acts thereafter. In order to develop this point as clearly as I can, let me first compare the Diana scenario with the following variation on the case.

Suppose, now, that Diana comes to the conclusion that the best way to ensure that Ernie will perform certain actions is to endow Ernie's zygote with certain properties and to continuously monitor and change Ernie's circumstances thereafter so that he will develop into an adult who will perform the right actions, at the right times, and on the basis of his own practical deliberations. One might find this scenario more worrying, initially at least, because here Diana monitors and changes the circumstances in Ernie's environment. However, what matters for my purposes is that this scenario helps us to see an important feature of the original Diana scenario. In the suggested variation, we can see how a process of continuous monitoring and manipulation of the circumstances could in fact guide Ernie's development and his actions in accordance with Diana's plan. In contrast, in the original Diana scenario, we are asked to assume that Diana can control Ernie's entire development and his behavior over a period of thirty years merely by arranging the initial properties of Ernie's zygote in a certain way. This, it should be noted, is truly and utterly incredible. During his development, Ernie will face an enormous amount of situational and highly complex social circumstances, and his intrinsic and dispositional properties 
will be subject to frequent change. In light of this, it is extremely difficult to see how Diana could possibly control his entire development and behavior by controlling only the initial properties of his zygote. As the set of properties carried by Ernie's zygote seems vanishingly small in comparison with the set of properties that he will acquire and that he will face during the thirty year period, it seems virtually impossible that one could control his interactions with the environment only by arranging the initial conditions of this highly complex process.

Of course, proponents of the argument can agree. Yes, what we are asked to imagine is incredible and perhaps practically impossible. But the Diana scenario is coherent and we assume, after all, that Diana has very special powers. Now, I do not want to challenge the use of the scenario for the argument, and my point does not concern its incredibility as such. Rather, my point here concerns the relation between the creation of the zygote and Ernie's development and behavior thereafter. Diana does not monitor and change the circumstances after the zygote is created (as in the suggested variation). Given this, it is clear that Diana could not ensure that Ernie will develop and act as planned by creating the zygote at some arbitrary time and place and into arbitrary circumstances. Rather, Diana must bring Ernie's zygote into circumstances that have, and will continue to have, exactly the right properties: circumstances that will interact with Ernie's intrinsic and dispositional properties as planned by Diana. Ernie's intrinsic and dispositional properties and the properties of the circumstances that he encounters must fit together - they must be made for each other.

Now we can see why the mentioned split between comparing the creation of the zygote, on the one hand, and considering Ernie's development and his actions, on the other, hides an important and problematic feature of the Diana scenario. If we consider the creation of the zygote and Ernie's subsequent development and behavior in isolation, we lose sight of the fact that those two parts of the story are rigged to fit together in the sense just explained. This is obviously a very striking difference, in comparison with normal deterministic scenarios. For all we know, the initial properties of zygotes and the circumstances in normal deterministic scenarios are not arranged so that they will interact at every step exactly as planned by another agent.

Does that mean that Ernie is manipulated? He is not manipulated in the sense that Diana does not change his intrinsic and dispositional properties after the zygote is created. He is also not manipulated in the sense that Diana does not change his circumstances so that he develops and acts in certain ways. We may express this by saying that Ernie's development, his 
circumstances, and his actions are not manipulated directly. But it does seem that his development, his circumstances, and all his actions are manipulated in the sense that the initial properties of his zygote are arranged so that they will interact with the circumstances as planned by Diana. Let us say that Ernie is, in this sense, manipulated indirectly. It is clear that agents in normal deterministic worlds are not manipulated in this way - at least not in virtue of the fact that they are agents in deterministic worlds.

According to Mele, the Diana scenario is not a manipulation scenario, because one can manipulate an individual only if that individual already exists. Ernie's zygote is created from scratch, as it were, which is why Mele calls the argument an "original-design" argument (2008: 284-285). Kearns (2012:381) and Todd (2013: 200) disagree. To them, it seems clear that there is at least a sense in which Ernie is manipulated: it seems clear that he is designed or preprogrammed to act in accord with Diana's plan. Their intuition is in line with my claim that Ernie is manipulated indirectly.

Now, it does not matter whether we decide to call the Diana scenario a "manipulation" or an "original-design" scenario. It does not even matter whether the Diana scenario is a manipulation or an original-design scenario. What matters, rather, is whether there is a difference between the Diana scenario and normal deterministic scenarios concerning freedom and moral responsibility. I have argued that the Diana scenario features a kind of indirect manipulation that is not present in normal deterministic scenarios. Generally, we tend to assume that a difference concerning manipulation makes a difference concerning freedom and moral responsibility, because we tend to assume that manipulation undermines freedom and moral responsibility. But there are, arguably, kinds of manipulation that are benign in the sense that they do not undermine freedom and moral responsibility. To see this, compare the following two cases.

Eduard is another agent in Diana's universe. His zygote came to exist in a normal way and he developed in a normal way, without Diana's intervention. At some point, however, Diana decides to arrange Eduard's circumstances so that he will perform certain actions. Diana knows, for instance, that Eduard will make and break a certain promise in a certain set of circumstances and she decides to arrange those circumstances. Compare this scenario with a case in which Eduard encounters the same circumstances as a matter of contingent happenstance and in which he makes and breaks the promise all the same. Does the fact that Diana arranged the circumstances in the first scenario make a significant difference? Does it render Eduard not free 
and not responsible? In light of the comparison, one may think that the difference is clearly not significant: it seems, at least, that if Eduard is free and responsible in the second scenario, then he is free and responsible in the first one as well. Moreover, one may think that this point generalizes. One may think, that is, that the fact that an agent's circumstances have been arranged by another agent never makes a significant difference concerning freedom and moral responsibility.

This generalization, however, is too quick. Let us grant that Diana's interference does not make a significant difference in the described promise scenario. Let us also grant that this point generalizes to most of Eduard's actions during adulthood. Obviously, from this it does not follow that the manipulation of an agent's circumstances never makes a significant difference. To investigate this further, let me distinguish between three different types of circumstances. First, some circumstances are formative: they shape the development of the self in ways for which one is clearly not responsible. Many of the circumstances that one encounters during childhood are of this kind. Second, some circumstances are transformative: they lead to reconfigurations of the self for which one is at least partly responsible. Some circumstances during adolescence and adulthood are transformative in this sense. Third, some circumstances are eliciting: they are circumstances in which one merely enacts or expresses one's character or personality traits.

It seems that the circumstances in Eduard's case are eliciting, because it seems that he is just the kind of person who would make and break a promise when put in those circumstances. This is, in part, why it seems clear that it does not make a significant difference whether those circumstances are arranged or not. Further, it seems that this point generalizes to most actions, because most of our actions are, arguably, elicited. Once we have developed a certain character with certain personality traits, we often merely enact or express those traits in the relevant circumstances. Given that those circumstances merely elicit those traits, it does not seem to matter whether or not those circumstances have been arranged or not.

Now, I do not want to deny the possibility that Eduard's circumstances are transformative, rather than eliciting. It is certainly conceivable that, by making and breaking the promise, Eduard makes a contribution to a transformation of his self for which he is at least partly responsible. Does it make a significant difference if such transformative circumstances are arranged by Diana? In this case, the answer is not obvious. A lot, I think, depends now on the further details of the case. However, we do not have to pursue this further, because the important question is a 
different one. The important question is whether the point generalizes: Is it the case that it never makes a significant difference whether or not an agent's circumstances have been arranged?

To bring this question into focus, suppose now that Diana arranges all the eliciting, all the formative, and all the transformative circumstances that Eduard encounters and compare this with a case in which he encounters exactly the same circumstances as a matter of contingent happenstance. Does it make a significant difference that Diana arranges all those circumstances so that Eduard will perform certain actions? My intuition is that this clearly does make a significant difference concerning freedom and moral responsibility. It would not matter if all eliciting circumstances were arranged. Perhaps it would not matter if, in addition, some formative and transformative circumstances were arranged - whether this would matter depends, again, on the further details of the case. But it does, I think, clearly matter if all the formative and transformative circumstances are arranged. My intuition, at least, is that if all the circumstances are arranged so that he will perform certain actions, then he is not free and not morally responsible. The same line of reasoning applies, mutatis mutandis, to Ernie.

In the Diana scenario, the initial properties of the zygote are rigged to interact with the circumstances so that the agent will develop and act exactly as planned. This is not the case in normal deterministic scenarios. I have suggested that this difference is a difference concerning indirect manipulation. More importantly, I have argued that it is a significant difference: Ernie does not seem to be free and morally responsible because Diana arranges the initial properties of his zygote to fit with all the circumstances that he will encounter, whereas agents in normal deterministic scenarios may well be free and responsible because they are not subject to this kind of indirect manipulation. On the basis of this we can conclude, then, that premise 2 of the zygote argument does not hold. ${ }^{3}$

\footnotetext{
${ }^{3}$ In two recent articles, Repko Waller (2014) and Barnes (forthcoming) reject premise 2 as well. I was not aware of their replies when I wrote this paper and I would like to thank an anonymous referee for the references. Their objections to premise 2 differ substantially from the reply that I just gave. According to Barnes, there is a significant difference between the Diana scenario and normal deterministic scenarios concerning the agent's potential for creativity. As we have seen, my response does not appeal to the notion of creativity at all. Repko Waller focuses on Diana's "effective intentions" in the creation of Ernie's zygote, and her objection is based on a general principle concerning blameworthiness. I have focused instead on the relation between the creation of the zygote and the circumstances that shape Ernie's development thereafter, and the mentioned principle on blameworthiness plays no role in my reply.
} 


\section{The dialectic and a dilemma}

I have just argued that reflection on the Diana scenario shows that premise 2 of the zygote argument does not hold. Recently, Fischer (2011) offered a thought experiment which suggests, as he argued, that we should reject premise 1 . In connection with that, it is worth noting that a rejection of premise 2 is far more effective in the defense of compatibilism. Fischer himself pointed out that:

$[\ldots]$ it is open to the proponent of the notion that the Zygote Argument increases the price of compatibilism to point out that it shows that an ordinary situation is just as worrisome as a scenario that is indeed prima facie problematic (the Diana scenario). (2011:271)

However, this is open to the proponent of the zygote argument only if the opponent grants premise 2 (as Fischer does). Premise 2 says that there is no significant difference. This allows the proponent of the zygote argument to turn the tables immediately, in case the opponent denies premise 1. It opens compatibilism to the charge that there is no significant difference between normal deterministic worlds and, say, a world in which all agents are subject to the kind of indirect manipulation that is at work in the Diana scenario. For this reason, a rejection of premise 1 results in a "dialectical stalemate", according to Fischer (ibid.). A rejection of premise 2, however, blocks not only the conclusion. It blocks also the possibility that the proponent of the argument can simply turn the tables against compatibilism in this way, and it achieves therefore considerably more than just a dialectical stalemate.

Kearns (2012) recently argued that reflection on the interaction between the two premises shows that manipulation arguments face, in general, a dilemma (388-389). In broad outline, this dilemma goes as follows. Take any deterministic manipulation scenario. Either the manipulation plays a role in making it the case that the agent is not free and not responsible, or it does not. ${ }^{4}$ If it does, then determinism alone does not make it the case that the agent is not free and not responsible, and so the scenario cannot establish incompatibilism. If it does not, then it should be possible to describe deterministic scenarios in which it is intuitively clear that it is the determinism alone that makes it the case that the agent is not free and not responsible. It is,

\footnotetext{
${ }^{4}$ To be more precise, one could distinguish here between a necessary and a sufficient role in making this the case. However, for my purposes, the offered formulation is precise enough.
} 
however, very unlikely that this can be achieved. Reference to deterministic cases that do not involve any manipulation would clearly beg the question, and cases that do involve manipulation always seem to undermine freedom and responsibility because they involve manipulation. In order to substantiate this last claim, Kearns asks us to consider failed attempts of manipulation in deterministic worlds (2012: 387).

Consider, for instance, a deterministic scenario in which Diana gets things right only insofar as Ernie develops into a properly reason-responsive and self-controlled agent. After that, he begins to perform many intentional actions that Diana did neither plan nor foresee, and he does not perform the action $A$ when he is thirty years old. Ernie, as we might say, started to act on his own. Agnostics about the compatibility question may very well judge, here, that Ernie is free and responsible, despite the fact that all his actions are determined. The reason for this would seem to be that the manipulation is largely unsuccessful. This suggests, in turn, that what undermines the agent's freedom and responsibility in the Diana scenario (and in other manipulation cases) is not the fact that the agent is determined, but the fact that the manipulation is successful (and of a certain kind, perhaps).

In response, Todd (2013) argued in a recent defense of the zygote argument that this way of presenting the issue overlooks a crucial distinction:

According to the proponent of the argument [...], the 'addition' of Diana to a 'normal' scenario involving Ernie can be relevant to bringing out the judgment that Ernie is not responsible. However, this is not to say that the proponent of the argument says that the 'addition' of Diana to such a scenario is itself relevant to Ernie's responsibility. (195)

So, the crucial distinction is a distinction between bringing out the intuitive judgment that the agent is not free and not responsible and actually making it the case that the agent is not free and not responsible. Concerning the dilemma, Todd goes on to say that:

[...] the proponent of the argument should admit that the manipulation does no work in making the agent unfree. Rather, the proponent of the argument contends - and clearly must contend - that the manipulation is irrelevant as concerns what makes the agent unfree. She instead says that the manipulation can help us see that something does make the agent unfree. (202) 
It seems, however, that something has gone wrong here. When used in arguments for incompatibilism, manipulation scenarios are supposed to show that there is no significant difference between being manipulated and being causally determined. From this, we are supposed to conclude that freedom and moral responsibility are incompatible with determinism, because freedom and moral responsibility are incompatible with manipulation. If, however, the part of a scenario that brings out the intuitive judgment that the agent is manipulated does not actually make it the case that the agent is not free and not responsible, then we should not jump to the conclusion that the agent is not free and not responsible, because we have not been given any good and independent reason to think that the other part of the scenario makes it the case that the agent is not free and not responsible. In fact, this line of argument generates a similar dilemma.

Consider a scenario that consists of a normal deterministic part and an added part, and suppose that the added part evokes the intuitive judgment that the agent is manipulated. Either the added part plays a role in making it the case that the agent is not free and not responsible, or it does not. If it does, then determinism alone does not make it the case that the agent is not free and not responsible, and so the scenario cannot establish incompatibilism. If it does not, then we should not jump to the conclusion that the agent is not free and not responsible, because we have not been given any good and independent reason to think that the other part of the scenario makes it the case that the agent is not free and not responsibility. Either way, the argument fails.

Let us call this the alternative dilemma. The proponent of the manipulation argument might reply that the argument contained in the second horn is too quick. Given that the scenario does evoke the intuitive judgment that the agent is not free and not responsible, and given that the added part does not do any work in making this the case, there is reason to think that the other part- that is, the normal deterministic part—must do all the work in making this the case.

However, should the claim that the very same part that brings out the intuitive judgment does actually not make it the case (that the agent is not free and not responsible) not give us pause and reason to question our prima facie intuition (that the agent is not free and not responsible)? I think it should, and I think that we should not, as I have put it, jump to the conclusion that the agent is not free and not responsible, because we have not been given any good and independent reason to think that the other part of the scenario does make it the case that the agent is not free and not responsible. Todd says that the suggested line of argument "may not be ultimately convincing to the compatibilist", but that it may well convince the agnostic (2013:202). He is 
certainly right about the former, and the latter is an open question. I would suggest that the agnostic may just as well be thoroughly confused and thrown into hesitation by the claim that the part of the scenario that brings out the intuitive judgment plays actually no role in making it the case (that the agent is not free and not responsible).

Further, it seems rather odd to hold that the addition of the story about how Diana creates the zygote brings out the intuitive judgment that the agent is not free and not responsible without making this the case. Why should we assume that the part that brings out the intuitive judgment does not make it the case? In particular, why should opponents share this assumption? If we add this assumption, then it follows that the other part must make it the case (provided, of course, that it $i s$ the case that the agent is not free and not responsible). By hypothesis, the other part is a normal deterministic part, and so Todd asks us, in effect, to add an assumption that yields incompatibilism. In other words, Todd asks us to add an assumption that begs the question.

My main response, however, is that the whole line of argument—which includes Todd's reply to Kearns and the stated objection to the second horn of the alternative dilemma-commits the same mistake as the argument for premise 2, discussed in section 2 . It hides a significant feature of the Diana scenario by asking us to split the agent's history into two parts and to consider them separately: it asks us to consider the way in which the zygote is created in isolation from the way in which the agent develops and acts thereafter. As before, we are told that one of the two parts does not make it the case that the agent is not free and not responsible, and it is suggested, as before, that the other part must therefore make this the case. But as before, this is too quick, because the kind of manipulation that is at work in the Diana scenario stems from the way in which the two parts are related, as I have argued at length in section 2.

Given this, we can reject Todd's reply to Kearns and we can reject the objection to the second horn of the alternative dilemma, because both are based on a spurious isolation of the two parts of the scenario. But for the very same reason, we should reject the alternative dilemma as well. The original dilemma stands, however, and so we can conclude, here, that manipulation arguments against compatibilism do indeed face a dilemma.

Manipulation arguments have generated a lot of debate, and so one might wonder why others have overlooked this dilemma. One possible explanation is that they have paid too much attention to the fact that there is no significant difference between the scenarios in terms of common compatibilist conditions. It is a mistake, as I have argued in section 2 , to think that 
opponents of manipulation arguments are restricted in their response by the conditions of common compatibilist theories. In the case of the zygote argument, one can reject premise 2 on the ground that there is a significant difference concerning freedom and moral responsibility. The question of whether or not this difference can be captured by current compatibilist theories is a further and separate issue. This means, though, that the zygote argument may be taken to show that contemporary accounts of compatibilism need to be revised or supplemented with a condition that excludes the kind of indirect manipulation exemplified by the Diana scenario. Doing so would not be ad hoc, I think. Compatibilists and incompatibilists have always agreed that manipulation tends to undermine freedom and moral responsibility. We have seen that the manipulation of circumstances may be benign. But we have also seen that the indirect manipulation at play in the Diana scenario is not benign. Given, then, that the indirect manipulation of Ernie in the Diana scenario does undermine freedom and moral responsibility, there would be nothing ad hoc about revising or supplementing a compatibilist theory with the aim of excluding this kind of indirect manipulation.

\section{Conclusion}

The zygote argument is the strongest manipulation argument against compatibilism that is currently on offer. Premise 2 of this argument says that there is no significant difference between the Diana scenario and normal deterministic scenarios. It does indeed seem that there is no way around this premise, as long as one considers the creation of the zygote in isolation from the circumstances and from the agent's further development. I have argued that this is a mistake. When we consider the question of whether there is a significant difference, we should also consider the creation of the zygote in relation to the circumstances and the agent's further development. Once we take this into account, we can see that there is a significant difference. The properties of Ernie's zygote are rigged to interact with the circumstances in accordance with Diana's plans. Agents in normal deterministic scenarios are not manipulated in this way. This shows, as I have argued, that premise 2 does not hold. Further, we have seen that a rejection of premise 2 is more effective in the defense of compatibilism than a rejection of premise 1, and I have argued that my objection to premise 2 helps to defend the claim that manipulation arguments face a dilemma. 


\section{References}

Barnes, E. C. forthcoming. Freedom, creativity, and manipulation. Noûs.

Dennett, D. C. 1984. Elbow room: The varieties of free will worth wanting. Cambridge, MA: MIT Press.

Fischer, J. M. 2011. The zygote argument remixed. Analysis 71: 267-272.

Fischer, J. M., \& Ravizza, M. 1998. Responsibility and control: A theory of moral responsibility. Cambridge: Cambridge University Press.

Frankfurt, H. 1971. Freedom of the will and the concept of a person. Journal of Philosophy 68: $5-20$.

Kane, R. 1996. The significance of free will. Oxford: Oxford University Press.

Kearns, S. 2012. Aborting the zygote argument. Philosophical Studies 160: 379-389.

Mele, A. 2006. Free will and luck. Oxford: Oxford University Press.

Mele, A. 2008. Manipulation, compatibilism, and moral responsibility. Journal of Ethics 12: $263-286$.

Pereboom, D. 2001. Living without free will. Cambridge: Cambridge University Press.

Repko Waller, R. 2014. The threat of effective intentions to moral responsibility in the zygote argument. Philosophia 42: 209-222.

Todd, P. 2013. Defending (a modified version of) the zygote argument. Philosophical Studies 164: 189-203. 